\title{
A CERTAIN TYPE OF CONTINUOUS CURVE AND RELATED POINT SETS*
}

\author{
BY \\ P. M. SWINGLE
}

Definition. A continuous curve every subcontinuum of which is a continuous curve will be called a perfect continuous curve. $\dagger$ In this paper, among other things, a study is made of this type of continuous curve.

An important problem in analysis situs is to determine whether a given point set is arc-wise connected. It is known that a connected subset of a perfect continuous curve is not necessarily arc-wise connected. $\ddagger$ Here, however, it is shown in Theorem 5 that, if $a$ and $b$ are two points of a connected subset $N$ of a perfect continuous curve, then the set common to $N$ and the arcs $a b$ of $N^{\prime}$ is connected. And in Theorem 7 it is shown that if $N^{\prime}$ contains but a countable number of $\operatorname{arcs} a b$ then $N$ contains an arc $a b . \S$

A generalization of the problem as to whether there exists an arc joining two points in a given point set is the following: when do there exist in a given point set where $q$ is a given positive integer, $q$ arcs, distinct except for end points, joining two given points; or still a further generalization would be to ask when do there exist $q$ such arcs joining two distinct closed point sets? This problem is considered in $\S \mathrm{II}$ of this paper. It might also be asked when do there exist $q$ distinct arcs joining two distinct closed point sets? This problem is considered in §I.

I wish to express my thanks and to acknowledge my great indebtedness to Professor R. L. Wilder for his suggestions, criticisms, and constant encouragement.*

* Presented to the Society, April 6, 1928; received by the editors in June, 1929, and June, 1930. Because of the delay in publishing this paper the privilege has been taken of using some recent results of other authors to abbreviate proofs and to obtain more general results than were originally obtained with respect to space and boundedness. These changes have been made in footnotes and in Section $\mathrm{V}$ with the exception that recent results have been used in proving Theorem 7 . In general proofs which are well known in recent papers have been omitted here.

$\dagger$ In this paper a connected im kleinen continuum will be called a continuous curve. For definitions see R. L. Moore, Report on continuous curves from the viewpoint of analysis situs, Bulletin of the American Mathematical Society, vol. 29 (1923), pp. 289-302.

$\ddagger$ B. Knaster and C. Kuratowski, $A$ connected and connected im kleinen point set which contains no perfect subset, Bulletin of the American Mathematical Society, vol. 33 (1927), pp. 106-109.

$\S$ For theorems on arc-wise connected subsets of a perfect continuous curve see R. L. Wilder: Characterizations of continuous curves that are perfectly continuous, Proceedings of the National Academy of Sciences, vol. 15 (1929), pp. 614-621, Theorem 3; Concerning perfect continuous curves, Proceedings of the National Academy of Sciences, vol. 16 (1930), pp. 233-240, Theorems 1 and 2. 


\section{Distinct arcs in a point Set}

In this section the following problem will be considered: when can it be said that in a set $M$, for any given positive integer $q$, either there exist $q$ distinct arcs $\dagger$ of $M$ joining two distinct closed point sets $\ddagger A$ and $B$ or there exist $q-1$ points such that every arc of $M$ joining $A$ and $B$ contains at least one of these points? If $M$ contains no arc joining $A$ and $B$ it will be understood that there exist $1-1=0$ points contained in every arc of $M$ joining $A$ and $B$. With this understanding it will be shown that one of the two above possibilities holds for every set $M$.

LEMMA 1. If $(t)$ is a set of arcs contained in an arc ab such that every point of $a b$ except $a$ and $b$ is an interior point of an arc of $(t)$ and both $a$ and $b$ are end points of at least one arc of $(t)$, then there exists a simple chain of arcs $\S$ of $(t)$ joining $a$ and $b$.

This follows in a manner very similar to that used by R. L. Moore in proving Theorem 10 of his paper On the foundations of plane analysis situs.||

LEMma 2. In $E_{n}$ T let $C$ and $K$ be distinct, closed, and bounded point sets, where $K$ is connected and does not separate $E_{n}$, and let $a_{i} w_{i}(i=1,2, \cdots, m)$ be $m$ distinct arcs such that $a_{i} w_{i} \times K=w_{i}$. Then there exists a positive number $d$ such that for any positive number $r$ less than $d$ there exist $m$ distinct arcs $a_{i} u_{i}$ of $a_{i} w_{i}$ and $m$ arcs $u_{i} h$ distinct except for $h$ such that (1) every point of $u_{i} h$ is at a distance less than $r$ from $K,(2)$ the arc $w_{i} u_{i}$ of $a_{i} w_{i}$ has nothing in common with $C$, and (3) for any $j, u_{j} h \times\left(\left(a_{i} u_{i}\right)^{* *}+C\right)=u_{j} h \times u_{j} a_{j}=u_{j}$.

* Since the theorems of this paper were proved a paper has appeared giving an interesting result in this connection: see N. E. Rutt, Concerning the cut points of a continuous curve where the arc curve, $A B$, contains exactly $N$ independant arcs, American Journal of Mathematics, vol. 51 (1929), p. 218. For another interesting result see W. L. Ayres, Concerning continuous curves in metric space, ibid., pp. 577-594, Theorem 6. See also K. Menger, Zur allgemeinen Kurventheorie, Fundamenta Mathematicae, vol. 10, p. 100, Theorem $\beta$. In each of these papers the $q$ arcs considered lie in a continuous curve in contrast to the results of this paper where the containing set may not even be connected in Theorem 1 and in Theorem 3 is connected but not necessarily closed.

$\dagger$ Two point sets are distinct when they have no common point.

$\ddagger$ An arc $a b$ will be said to join the point sets $A$ and $B$ if $A \times a b=a$ and $B \times a b=b$. Under these conditions the arc $b a$ will be said to join $B$ and $A$.

$\S$ If $a$ and $b$ are distinct points, then a simple chain from $a$ to $b$ is a finite sequence of arcs $t_{i}(i=1,2, \cdots, m)$ such that (1) $t_{i}$ contains $a$ if and only if $i=1,(2) t_{i}$ contains $b$ if and only if $i=m$, and (3) if $1 \leqq i \leqq m$ and $1 \leqq j \leqq m, i<j$, then $t_{i}$ has a point in common with $t_{j}$ if and only if $j=i+1$.

\| These Transactions, vol. 17 (1916), pp. 131-164.

T The notation $E_{n}$ will be used to denote a euclidean space of $n$ dimensions.

** Throughout this paper the set $a_{1}+a_{2}+\cdots+a_{m}$ will be designated by $\left(a_{i}\right)(i=1,2, \cdots, m)$. Thus $\left(a_{i}\right)+\left(b_{i}\right)=a_{1}+a_{2}+\cdots+a_{m}+b_{1}+b_{2}+\cdots+b_{m}$. The range of values for $i$, if it is not given, will refer to the last mentioned range of values for $i$. 
The proof will first be given for $E_{2}$ as this is the most difficult case. Let $z_{i}$ be the first point of $C+a_{i}$ on $w_{i} a_{i}$ and let $a_{i} z_{i}=a_{i}$ if $z_{i}=a_{i}$. Let $d$ be a positive number such that every point of $C+\left(a_{i} z_{i}\right)$ is at a distance greater than $d$ from $K$. Let $M=K+C+\left(a_{i} z_{i}\right)$. Then for any positive number $r$ less than $d$ there exists* a simple closed curve $J$ bounding a region $R$ such that $J \times M=0$, $R \times M=K$, and every point of $R$ is at a distance less than $r$ from $K$. Let the first point of $J$ on $a_{i} w_{i}$ be $u_{i}$, and let $a_{i} u_{i}$ be an arc of $a_{i} w_{i}$. Let $h$ be any point of $R$. Then there exist in $R$ the $m$ arcs $h u_{i}$, distinct except for $h$, which together with the arcs $a_{i} u_{i}$ have the properties described in this theorem.

For $E_{n}, n>2$, a connected domain $R$ can be obtained by means of a finite set of spheres of radius $r$ and covering $K$. The proof is then similar to the above.

It will simplify the proof of the main theorem of this section to prove first the following lemma.

Lемма 3. Suppose for the integer $k$ it is true that for every point set $G$, either there exist $k-1$ points such that any arc of $G$, joining any two distinct closed point sets, contains at least one of these points or there exist $k$ distinct arcs of $G$ joining these two distinct closed point sets. Then in $E_{n}$ let $a_{i} b_{i}(i=1,2, \cdots$, $k$ ) be $k$ distinct arcs joining two distinct closed point sets $A$ and $B$ and $A_{i} B_{i}$ be $k$ distinct arcs such that (1) $A$ contains $\left(A_{i}\right) \times(A+B)$ and $B$ contains $\left(B_{i}\right)$ $\times(A+B),(2) A_{i} B_{i} \times a_{1} b_{1}=A_{i}+B_{i}-\left(A_{i}+B_{i}\right) \times(A+B),(3) A_{i} B_{i} \times(A+B)$ contains at most $A_{i}+B_{i}$, and (4) $a_{1} A_{i}$ of $a_{1} b_{1}$ does not contain a point of $\left(B_{i}\right)$. Then there exist $k$ distinct arcs $w_{i}$ of $M=\left(a_{i} b_{i}\right)+\left(A_{i} B_{i}\right)$ joining $A$ and $B$ and there exists an arc $w_{k+1}=W_{1} W_{2}$ of $a_{1} b_{1}$, where $W_{1}$ is contained in $\left(A_{i}\right) \times a_{1} b_{1}+a_{1}$ and $W_{2}$ in $\left(B_{i}\right) \times a_{1} b_{1}+b_{1}$, such that $\left(w_{i}\right) \times w_{k+1}$ contains at most $W_{1}+W_{2}$, $a_{1} W_{1}$ of $a_{1} b_{1}$ does not contain $W_{2}$, and there exists an arc of $\left(w_{i}\right)+w_{k+1}$ which contains $b_{1} W_{2}$ of $a_{1} b_{1}$.

Consider for example the case where $A_{3}$ is the first point of $\left(A_{i}\right)+a_{1}$ on $b_{1} a_{1} \dagger$ and $B_{4}$ is the first point of $\left(B_{i}\right)+b_{1}$ on $a_{1} b_{1}$. Let $p_{i} q_{i}$ be an arc of $A_{i} B_{i}$ where $p_{i}$ precedes $q_{i}$ on $A_{i} B_{i}, p_{i} \neq A_{i}$, and $q_{i} \neq B_{i}$. Let $p_{i} B_{i}$ and $q_{i} A_{i}$ be arcs of $A_{i} B_{i}$ and let $a_{1} A_{3}$ and $b_{1} B_{4}$ be arcs of $a_{1} b_{1}$. Let $T_{a}$ be composed of $\left(p_{i} B_{i}\right)$ $+\left(a_{j} b_{j}\right)+b_{1} B_{4}(j=2,3, \cdots, k)$ and also of $q_{i} A_{i}$ if and only if $A$ contains $A_{i}$; let $T_{b}$ be composed of $\left(q_{i} A_{i}\right)+\left(a_{j} b_{j}\right)+a_{1} A_{3}$ and also of $p_{i} B_{i}$ if $B$ contains $B_{i}$. Let $d$ be a positive number such that no point of $T_{a}$ is at a distance less than $d$ from $a_{1} A_{3}$ and no point of $T_{b}$ is at a distance less than $d$ from $b_{1} B_{4}$.

* R. L. Moore, Concerning the separation of point sets by curves, Proceedings of the National Academy of Sciences, vol. 11, No. 8, pp. 469-476, Theorem 1.

$\dagger$ The first point of a set $C$ on an arc $a b$ is the first point going from $a$ to $b$; the first point of $C$ on $b a$ is the first point going from $b$ to $a$. 
The sets $T_{a}$ and $a_{1} A_{3}$ are distinct, closed, and bounded point sets where $a_{1} A_{3}$ is connected and does not separate $E_{n}$. Take $r$ less than $d / 3$. The arcs $q_{i} A_{i}$, which have a point common with $a_{1} b_{1}$, have the same properties with reference to $a_{1} A_{3}$ as the $\operatorname{arcs} a_{i} w_{i}$ in Lemma 2 have to $K$. Say that there are $m$ of these $\operatorname{arcs} q_{i} A_{i}$ which have a point common with $a_{1} b_{1}$. Then by Lemma 2 there exist $m$ arcs and also there exist $k-m$ vacuous point sets, giving a total of $k$ sets $h_{1} u_{1 i}$, where $h_{1} u_{1 i}$ is vacuous if $A$ contains $A_{i}$ and consists of an arc if $A$ does not contain $A_{i}$, and similarly there exist $k$ arcs or vacuous sets $B_{i} u_{1 i}$, where $B_{i} u_{1 i}$ is contained in $A_{i} B_{i}$. It is seen that the sets $B_{i} u_{1 i}+u_{1 i} h_{1}$ are distinct except for $h_{1}$, the set $\left(A_{i} u_{1 i}+u_{1 i} h_{1}\right) \times T_{a}=0$, where $A_{i} u_{1 i}=0$ or is an arc according as $A$ does or does not contain $A_{i}$, and every point of $h_{1} u_{1 i}$ is at a distance less than $r$ from $a_{1} A_{3}$. From the last mentioned property it follows that the new sets $h_{1} u_{1 i}$ are at a distance greater than $d / 3$ from $b_{1} B_{4}$. Then Lemma 2 can be again applied where $T_{b}+\left(h_{1} u_{1 i}\right)=C$ and $b_{1} B_{4}=K$ are the distinct, closed, and bounded point sets and the $\operatorname{arcs} B_{i} p_{i}$, which have a point common with $a_{1} b_{1}$, are the $m$ distinct arcs of that hypothesis. Thus a new set of $k$ arcs or vacuous point sets $h_{2} u_{2 i}$, distinct except for $h_{2}$, and a set of $k$ distinct arcs or vacuous point sets $p_{i} u_{2 i}$, where $p_{i} u_{2 i}$ is contained in $p_{i} B_{i}$ and so in $u_{1 i} B_{i}$, are obtained. There exists then a set of $k$ distinct $\operatorname{arcs} u_{1 i} u_{2 i}$ in $\left(A_{i} B_{i}\right)$ where $u_{1 i}=A_{i}$ if $A$ contains $A_{i}$ and $u_{2 i}=B_{i}$ if $B$ contains $B_{i}$. Thus a set of $k$ arcs $h_{1} u_{1 i}+u_{1 i} u_{2 i}+u_{2 i} h_{2}=t_{i}$, distinct except for $h_{1}$ and $h_{2}$, are obtained where $\left(h_{1} u_{1 i}+u_{2 i} h_{2}\right)$ is at a distance less than $r$ from $a_{1} A_{3}+B_{4} b_{1}$ and so has no points in common with $\left(a_{j} b_{j}\right)$. Let $G=\left(a_{j} b_{j}\right)+t_{i}$.

Suppose that there exists a set $H$ consisting of $k-1$ points such that every arc of $G$ joining $A+h_{1}$ and $B+h_{2}$ contains at least one point of $H$. Therefore there exists one of these points on each of the $k-1 \operatorname{arcs} a_{j} b_{j}$. Since then the points of $H$ are on $a_{j} b_{j}$ and since $\left(h_{1} u_{1 i}+u_{2 i} h_{2}\right) \times\left(a_{j} b_{j}\right)=0$, the set $H \times\left(h_{1} u_{1 i}+u_{2 i} h_{2}\right)=0$. Thus if there exists a point of $H$ on each of the $\operatorname{arcs} t_{i}$ joining $A+h_{1}$ and $B+h_{2}$ there must exist a point of $H$ on each of the $k$ distinct $\operatorname{arcs} u_{1 i} u_{2 i}$ of $t_{i}$. But as $H$ contains only $k-1$ points this is impossible. Therefore it is necessary under our hypothesis that $G$ contain $k$ distinct arcs, $v_{i}$ say, joining $A+h_{1}$ and $B+h_{2}$.

There exist three cases to consider having obtained the $k$ arcs $v_{i}$ : (I) the set $\left(v_{i}\right)$ contains $h_{1}+h_{2}$; (II) it contains one and only one of these points; and (III) it contains neither of them.

(I) Under this case there exist two possibilities: one arc of the set $\left(v_{i}\right)$ may contain both $h_{1}$ and $h_{2}$ or one arc may contain one of these points and another arc contain the other.

(a) Consider for example the case where $v_{1}$ contains $h_{1}+h_{2}$. Then $v_{1}$ con- 
tains a point of $\left(u_{1 i}\right), u_{12}$ say, and a point of $\left(u_{2 i}\right), u_{23}$ say. It will now be shown that $w_{1}=a_{1} A_{2}+A_{2} u_{12}+u_{12} u_{23}+u_{23} B_{3}+B_{3} b_{1}$ is such that $w_{1} \times\left(v_{j}\right)=0$, where $a_{1} A_{2}+B_{3} b_{1}$ is contained in $a_{1} b_{1}, A_{2} u_{12}$ in $A_{2} B_{2}, u_{23} B_{3}$ in $A_{3} B_{3}$, and $u_{12} u_{23}$ in $v_{1}$. Since $v_{1} \times\left(v_{j}\right)=0, u_{12} u_{23} \times\left(v_{j}\right)=0$. Since $v_{1}$ contains $h_{1}+h_{2}$, it is the only one of the arcs of $\left(v_{i}\right)$ which contains a point of $h_{1} u_{1 i}+h_{2} u_{2 i}$. Hence the set $\left(a_{j} b_{j}\right)+\left(u_{2 i} u_{1 i}\right)-\left(u_{1 i}\right)-\left(u_{2 i}\right)$ contains $\left(v_{j}\right)$. But the set $a_{1} b_{1} \times\left(\left(a_{j} b_{j}\right)+\left(u_{1 i} u_{2 i}\right)\right)$ $=0$ for $a_{1} b_{1} \times\left(a_{j} b_{j}\right)=0$ and as $a_{1} b_{1}$ contains at most $A_{i}+B_{i}$ of $A_{i} B_{i}, a_{1} b_{1}$ $\times\left(u_{1 i} u_{2 i}\right)=0$. Therefore $\left(a_{1} A_{2}+B_{3} b_{1}\right) \times\left(v_{j}\right)=0$. It remains to prove that $\left(A_{2} u_{12}+B_{3} u_{23}\right) \times\left(v_{j}\right)=0$. This follows from the fact that $\left(a_{j} b_{j}\right)+\left(u_{1 i} u_{2 i}\right)-\left(u_{1 i}\right)$ $-\left(u_{2 i}\right)$ contains $\left(v_{j}\right)$. Therefore $w_{1} \times\left(v_{j}\right)=0$. Let now $w_{j}=v_{j}$ and let $w_{k+1}$ $=A_{2} B_{3}$ of $a_{1} b_{1}$. As shown above, $a_{1} b_{1} \times\left(w_{j}\right)=0$ and so $w_{k+1} \times\left(w_{j}\right)=0$. And $w_{k+1} \times w_{1}=A_{2}+B_{3}$ while $w_{1}$ contains $b_{1} B_{3}$.

(b) Consider now for example the case where $v_{1}=h_{1} b$ and $v_{2}=h_{2} a$, where $A$ contains $a$ and $B$ contains $b$. Then $v_{1}$ contains a point, $u_{11}$ say, of $\left(u_{1 i}\right)$, and $v_{2}$ one, $u_{21}$ say, of $\left(u_{2 i}\right)$. Here $\left(a_{j} b_{j}\right)+\left(u_{1 i} u_{2 i}\right)-\left(u_{1 i}\right)-\left(u_{2 i}\right)$ contains $\left(v_{f}\right)+b u_{11}$ $+a u_{21}(f=3,4, \cdots, k)$, where $v_{1}$ contains $b u_{11}$ and $v_{2}$ contains $a u_{21}$. Thus $u_{11} A_{1}+A_{1} a_{1}+u_{21} B_{1}+B_{1} b_{1}$ does not contain a point of $\left(v_{f}\right)+b u_{11}+a u_{21}$ except $u_{11}$ and $u_{21}$, where $A_{1} B_{1}$ contains $u_{11} A_{1}+u_{21} B_{1}$, and $a_{1} b_{1}$ contains $a_{1} A_{1}+b_{1} B_{1}$. Let $w_{f}=v_{f}$, let $w_{1}=a_{1} A_{1}+A_{1} u_{11}+u_{11} b$, and let $w_{2}=b_{1} B_{1}+B_{1} u_{21}+u_{21} a$. Let $w_{k+1}$ $=A_{1} B_{1}$ of $a_{1} b_{1}$. Now $w_{1} \times\left(w_{f}\right)=w_{2} \times\left(w_{f}\right)=0$ and $w_{1} \times w_{2}=0$. Also $w_{1} \times w_{k+1}$ $=A_{1}$ and $w_{2} \times w_{k+1}=B_{1}, w_{k+1} \times\left(w_{f}\right)=0$ and $w_{2}$ contains $b_{1} B_{1}$ of $a_{1} b_{1}$.

(II) In considering the case where $\left(v_{i}\right) \times\left(h_{1}+h_{2}\right)$ contains one and only one point, there exist two cases according as $h_{1}$ or $h_{2}$ is the point contained.

(a) Consider for example the case where $v_{1}=h_{1} b$, where $B$ contains $b$, and say $v_{1}$ contains $u_{11}$. Let $w_{j}=v_{j}$ and let $w_{1}=b u_{11}+u_{11} A_{1}+A_{1} a_{1}$, where $b u_{11}$ is contained in $v_{1}, u_{11} A_{1}$ in $A_{1} B_{1}$, and $A_{1} a_{1}$ in $a_{1} b_{1}$. Let $w_{k+1}=A_{1} b_{1}$. Then the $k$ $\operatorname{arcs} w_{i}$ are distinct, $w_{k+1} \times\left(w_{j}\right)=0$, and $w_{k+1} \times w_{1}=A_{1}$.

(b) Consider now for example the case where $v_{1}=a h_{2}$, which contains $u_{23}$, say, where $A$ contains $a$. Let $w_{j}=v_{j}$ and let $w_{1}=a u_{23}+u_{23} B_{3}+B_{3} b_{1}$, where $a u_{23}$ is contained in $v_{1}, u_{23} B_{3}$ in $A_{3} B_{3}$, and $B_{3} b_{1}$ in $a_{1} b_{1}$. Let $w_{k+1}=a_{1} B_{3}$ of $a_{1} b_{1}$. Here $\left(w_{1}+w_{k+1}\right) \times\left(w_{j}\right)=0$ and $w_{1} \times w_{k+1}=B_{3}$ while the arc $w_{1}$ contains $b_{1} B_{3}$.

(III) For the case where $\left(v_{i}\right) \times\left(h_{1}+h_{2}\right)=0$ let $w_{i}=v_{i}$ and $w_{k+1}=a_{1} b_{1}$.

In this manner the lemma is proved for every possible case.

Lемма 4. Suppose for the integer $k$ it is true that for every point set $G$, either there exist $k-1$ points such that any arc of $G$, joining any two distinct closed point sets, contains at least one of these points, or there exist $k$ distinct arcs of $G$ joining these two distinct closed point sets. Then in $E_{n}$ let $a_{i} b_{i}(i=1,2, \cdots, k)$ be $k$ distinct arcs joining two distinct closed point sets $A$ and $B$, and ay an arc such that $A \times a y=a$ and $a_{1} b_{1} \times a y=y$ and $\left(a_{j} b_{j}\right) \times a y=0(j=2,3, \cdots, k)$; also 
let $A_{i} B_{i}$ be $k$ distinct arcs such that (1) $A$ contains $\left(A_{i}\right) \times(A+B)$ and $B$ contains $\left(B_{i}\right) \times(A+B)$, (2) $A_{i} B_{i} \times\left(a_{1} b_{1}+a y\right)=A_{i}+B_{i}-\left(A_{i}+B_{i}\right) \times(A+B)$, (3) $A_{i} B_{i} \times(A+B)$ contains at most $A_{i}+B_{i}$, (4) $a_{1} y$ of $a_{1} b_{1}$ and ay do not contain a point of $\left(B_{i}\right)$, and (5) $b_{1} y$ of $a_{1} b_{1}$ does not contain a point of $\left(A_{i}\right)$. Then there exist $k$ distinct arcs $w_{i}$ of $M=\left(a_{i} b_{i}\right)+\left(A_{i} B_{i}\right)+$ ay joining $A$ and $B$ and an arc $w_{k+1}=a_{0} x$, where $a_{0}$ is contained in $A$ and $x$ in $\left(B_{i}\right) \times a_{1} b_{1}+b_{1}$, and such that if there does not exist an arc $w_{i}$ containing $b_{1}$ then $w_{k+1}=a_{1} b_{1}$ or $w_{k+1}=a y+y b_{1}$ and $w_{k+1} \times\left(w_{j}\right)=0$, but if $w_{1}$, say, contains $b_{1}$ then $w_{1} \times w_{k+1}=x$ and $w_{k+1} \times\left(w_{j}\right)$ $=0$, and in every case $\left(w_{i}\right)+w_{k+1}$ contains $b_{1} y$ and there exists a $g$ such that $w_{0}$ contains $b_{1} x$ of $a_{1} b_{1}$.

The proof is similar to that of Lemma 3.

THEOREM 1. If $M$ is any point set in $E_{n}$, and $A$ and $B$ are any two distinct closed point sets, then, for any positive integer $q$, either there exists a point set $N$ containing $q-1$ points such that every arc of $M$ joining $A$ and $B$ contains at least one point of $N$ or there exist at least $q$ distinct arcs of $M$ joining $A$ and $B$.

If $q=1$, it is evident that the theorem is true. Assume that it is true for $q=k$. It will now be proved to be true for $q=k+1$.

There are two cases to consider according as either (I) there are $k-1$ points such that every arc of $M$ joining $A$ and $B$ contains at least one of these points, or (II) there are at least $k$ distinct $\operatorname{arcs}$ of $M$ joining $A$ and $B$.

(I) Consider the first case where the $k-1$ points exist. Then if any point is added to these, any arc of $M$ joining $A$ and $B$ contains at least one of these $k$ points. Hence in this case the theorem must be true for $q=k+1$ if it is for $q=k$.

(II) Consider now the case where there are at least $k$ distinct arcs of $M$ joining $A$ and $B$. Let $a_{i} b_{i}(i=1,2, \cdots, k)$ be $k$ such arcs. Consider any point $p$ of $a_{1} b_{1}$. Either (1) there is a set $N$ of $k-1$ points such that every arc of $M-p$ joining $A$ and $B$ contains a point of $N$ or (2) there are $k$ arcs of $M-p$ which are distinct and join $A$ and $B$.

(1) For the case where the set $N$ exists, $N+p$ is a set of $k$ points such that every arc of $M$ joining $A$ and $B$ contains at least one of these points. Thus in this case also if the theorem is true for $q=k$ it is for $q=k+1$.

(2) Consider now the remaining case where, for every point $p$ of $a_{1} b_{1}$, $M-p$ contains at least $k$ distinct arcs joining $A$ and $B$. For a certain point $p$ let $e_{i}(i=1,2, \cdots, k)$ be $k$ such distinct arcs. Take for example the case where $a_{1} \neq p \neq b_{1}$. For any $i$ there exists a region $R$ containing $p$ and so containing an $\operatorname{arc} t$ of $a_{1} b_{1}$ having $p$ as an interior point, such that $R^{\prime} \times e_{i}=0$. Let*

* If $M$ is a point set, $M^{\prime}$ will denote $M$ together with the limit points of $M$. 
$\left(a_{1} b_{1}-t\right)^{\prime}=t_{1}+t_{2}$. Then, if $t_{1}$ contains $a_{1}$, there exists an arc of $e_{i}, s_{i}$ say, joining $t_{1}+A$ and $t_{2}+B$. If $a_{1} b_{1}$ contains both end points of $s_{i}$, let $r_{i}$ be the arc of $a_{1} b_{1}$ joining these two points; if one of these end points is in $A$ and one in $a_{1} b_{1}$, let $r_{i}$ be the arc of $a_{1} b_{1}$ joining $a_{1}$ and the end point of $s_{i}$ in $a_{1} b_{1}$; if $B$ contains one of these end points and $a_{1} b_{1}$ contains the other, let $r_{i}$ be the arc of $a_{1} b_{1}$ joining $b_{1}$ and this end point in $a_{1} b_{1}$; and if $A$ contains one end point and $B$ contains the other, let $r_{i}=a_{1} b_{1}$. Thus to each arc $e_{i}$ there corresponds an $\operatorname{arc} r_{i}$ of $a_{1} b_{1}$ having $p$ as an interior point. Let $h$ be the $\operatorname{arc} r_{1} \times r_{2} \times \cdots$ $\times r_{k}$ of $a_{1} b_{1}$. Thus for each point $p$ of $a_{1} b_{1}$ there exists an arc $h$, having $p$ as an interior point unless $p=a_{1}$ or $p=b_{1}$ in which latter case $p$ is an end point, and a corresponding set, $f$ say, consisting of $k$ distinct $\operatorname{arcs} e_{i}$ joining $A$ and $B$ and having only end points of $h$ common with $h$. Let $(h)$ be the set of arcs such as $h$ and $(f)$ the set of sets such as $f$. Then by Lemma 1 there exists a simple chain, $h_{1}, h_{2}, \cdots, h_{m}$ of arcs of $(h)$ joining $a_{1}$ and $b_{1}$, where $h_{1}$ contains $a_{1}$ and $h_{m}$ contains $b_{1}$. Also there exists a set $f_{j}$ of $(f)$ corresponding to each $h_{j}$.

The set $f_{1}$ contains $k$ distinct arcs $A_{1 i} B_{1 i}$ joining $A$ and a closed subset of $a_{1} b_{1}-a_{1}+B$ where each $a_{1} B_{1 i}$ of $a_{1} b_{1}$ contains $h_{1}$, if $a_{1} b_{1}$ contains $B_{1 i}$. We have here, just as we had in the hypothesis of Lemma $3, k$ distinct $\operatorname{arcs} a_{i} b_{i}$ and a set of $k$ distinct $\operatorname{arcs} A_{1 i} B_{1 i}$. Applying this lemma we obtain a set of $k$ distinct $\operatorname{arcs} a_{1 i} b_{1 i}$ joining $A$ and $B$, which are the $\operatorname{arcs} w_{i}$ of that lemma, and an arc $a_{1} y_{1}$ of $a_{1} b_{1}$ joining $a_{1}$ and $\left(a_{1 i} b_{1 i}\right) \times\left(B_{1 i}\right)+B$, which is the arc $w_{k+1}$. The new set $\left(a_{1 i} b_{1 i}\right)+a_{1} y_{1}$ is contained in $\left(a_{i} b_{i}\right)+\left(A_{1 i} B_{1 i}\right)$. If $B$ contains $y_{1}$ then there exist $k+1$ distinct arcs of $M$ joining $A$ and $B$ provided $a_{1} y_{1}$ $\times\left(a_{1 i} b_{1 i}\right) \neq b_{1}$; and if $B$ does not contain $y_{1}$, as was shown in Lemma 3 , there exists an arc, $a_{11} b_{11}$ say, where $b_{11}=b_{1}$, containing $y_{1} b_{1}$ of $a_{1} b_{1}$. Thus either there exist $k+1$ distinct arcs of $M$ joining $A$ and $B$ or there exist $k+1 \operatorname{arcs}$ of $M$ joining $A$ and either $B+b_{1}$ or $B+b_{1} y_{1}$, which are distinct, with the exception that two of them contain a common end point in $b_{1} y_{1}$, and so in some $h_{i}$, $j>1$. If this end point is not in $h_{m}$, say for example that it is an interior point in $h_{2}$. We now have $k$ distinct $\operatorname{arcs} a_{1 i} b_{1 i}$, similar to the $\operatorname{arcs} a_{i} b_{i}$ of Lemma 4, and an arc $a_{1} y_{1}$ similar to $a y$. And there exist in $f_{2} k$ distinct $\operatorname{arcs} A_{2 i} B_{2 i}$, similar to $A_{i} B_{i}$ of this lemma, joining $A+a_{1} y_{1}+x_{a}$ and $B+x_{b}$, where $\left(a_{11} b_{11}\right.$ $\left.-h_{2}\right)^{\prime}=x_{a}+x_{b}$, and $x_{b}$ contains $y_{1} b_{1}$ of $a_{1} b_{1}$. Thus by this lemma it follows that there exist $k$ distinct $\operatorname{arcs} a_{2 i} b_{2 i}$ joining $A$ and $B$ and an $\operatorname{arc} a_{02} y_{2}$ joining $A$ and $\left(B_{2 i}\right) \times\left(a_{2 i} b_{2 i}\right)+B$. These $k+1$ arcs are distinct or else a new set is obtained, proceeding in the same manner as above, by the use of Lemma 4.

It is necessary then that either $k+1$ distinct arcs joining $A$ and $B$ be obtained or there exist an arc $a_{00} y_{0}$ such that $h_{m}$ of $a_{1} b_{1}$ contains $y_{0}$. Hence, by one further application of Lemma $4, k+1$ distinct arcs joining $A$ and $B$ 
must be obtained. Thus if the theorem is true for $q=k$ it is true for $q=k+1$ in this case.

In every case the theorem is true for $q=k+1$ if it is true for $q=k$. The theorem is true for $q=1$. Hence it is true for any value of $q$.

Corollary 1. If $M$ is a continuous curve in $E_{n}$ and $A$ and $B$ are any two distinct closed point sets, then either there exist, for any $q$, at least $q$ distinct arcs of $M$ joining $A$ and $B$, or there exists a point set $N$ of $q-1$ points such that $M-N$ does not contain a connected subset which contains points of both $A$ and $B$.

\section{ARCS, DISTINCT EXCEPT FOR END POINTS}

Here the following problem will be considered: when can it be said that in a set $M$ either there exists a set of at least $q$ arcs, where $q$ is any positive integer, distinct except for possibly their end points, joining two distinct closed point sets $A$ and $B$, or there exists a set $N$ of $q-1$ points, contained in $M-A-B$, such that every arc of $M$ joining $A$ and $B$ contains at least one point of $N$ ? Here also it will be understood that if $M$ contains no arcs joining $A$ and $B$, then the set $N$ is vacuous. A complete solution of the above problem is not obtained in this paper.

In proving the next theorem the following lemma is useful and is stated here without proof*

Lemma 5. If, in $E_{2}, M$ is a continuous curve which contains a subcontinuum which is not a continuous curve and if $k$ is any positive integer, then $M$ contains $k$ distinct arcs $a_{i} b_{i}(i=1,2, \cdots, k)$, and a sequence of distinct arcs $x_{j} y_{j}$ $(j=1,2, \cdots)$ having a sequential limiting set $Z$, such that $x_{j} y_{j} \times a_{i} b_{i} \not \neq 0$, $x_{j} y_{j} \times Z=0, x_{j} y_{j} \times a_{1} b_{1}=x_{j}$, and $x_{j} y_{j} \times a_{k} b_{k}=y_{j}$. Furthermore $x_{j}$ precedes $x_{j+1}$ on $a_{1} b_{1}, y_{i}$ precedes $y_{j+1}$ on $a_{k} b_{k}$, every point of $a_{i} b_{i}$ precedes every point of $a_{i+1} b_{i+1}$ on $x_{j} y_{j}, a_{i} b_{i} \times Z=b_{i}$, and $a_{i} b_{i} \times x_{1} y_{1}=a_{i}$.

THEOREM 2. Let $q$ be a given positive integer greater than one. Then in order that a continuous curve $M$, in $E_{2}$, be perfect, it is sufficient, if $L$ is the point set consisting of the points of any set of arcs of $M$ joining any two distinct closed point sets $A$ and $B$, that either there exist a set $N$ of $q-1$ points, of $L-A-B$, such that every arc of $L$ joining $A$ and $B$ contains at least one point of $N$, or there exist at least $q$ arcs of $L$, distinct except for possibly their end points, joining $A$ and $B$.

Assume that $M$ contains a subcontinuum which is not a continuous curve. Then by applying Lemma 5 the arcs of the conclusion there are obtained,

* The proof follows from the work of R. L. Wilder, Fundamenta Mathematicae, vol. 7, pp. 362-363, and from Theorem XXI by H. Hahn, Wiener Sitzungsberichte, vol. 123 (Part IIa), p. 2475. 
taking $k=q+1$. On the arc $x_{i} y_{j}$ there obtained, taking $j=2 t-1(t=1,2, \cdots)$, let $u_{t}$ be the subarc joining $a_{1} b_{1}$ and $a_{3} b_{3}$; and taking $j=2 t$ let $v_{t}$ be the subarc of $x_{i} y_{j}$ joining $a_{1} b_{1}$ and $a_{2} b_{2}$. Let $w_{t}$ be the subarc of $a_{2} b_{2}$ joining $v_{t}$ and $u_{t+1}$; and let $z_{t}$ be the subarc of $a_{1} b_{1}$ joining $u_{t}$ and $v_{t}$. Let $A=\left(a_{i}\right)$ and $B=Z$. Then every point of $L=\left(u_{t}+v_{t}+w_{t}+z_{t}\right)+a_{3} b_{3}+a_{4} b_{4}+\cdots+a_{q+1} b_{q+1}$ is contained in an arc of $L$ joining $A$ and $B(t=1,2, \cdots)$. But as $\left(u_{t}+v_{t}+w_{t}+z_{t}\right)$ does not contain an arc of $L$ joining $A$ and $B, L$ contains at most $q-1$ arcs, distinct except for possibly their end points, joining $A$ and $B$. However it is evident that $L-A-B$ does not contain $q-1$ points such that every arc of $L$ joining $A$ and $B$ contains at least one of these points. As this is a contradiction with our hypothesis, $M$ must be perfect.

Whether this condition is also necessary is not determined in this paper, except for $q=2$.

The proof of the following lemma can be obtained by means of a theorem by H. M. Gehman.*

LeMma 6. If $M$ is a bounded continuous curve in $E_{2}$, $\dagger$ then a necessary (and sufficient) condition that $M$ be perfect is the following: let $Z$ be any closed subset of $M, W$ any subset of $M$ such that $Z \times W=0$ and every point of $W$ can be joined to $Z$ by an arc contained in $W$ except for an end point in $Z$, and $x$ a limit point of $W$ such that $(W+Z) \times x=0$. Then there exists an arc joining $x$ and $Z$ which is contained in $W$ except for its end points.

THEOREM 3. If $q=2$ then the condition in Theorem 2 is also necessary, if $M$ is bounded. $\ddagger$

Suppose that there exist sets $L, A$, and $B$ such that there does not exist a point which is contained in $L-A-B$ and in every arc of $L$ joining $A$ and $B$. Then there exists an arc $a b$ joining $A$ and $B$. It then follows from the above theorem by H. M. Gehman and from Lemma 6 that for any point $p$ of $a b$, except possibly $a$ and $b$, there exists an arc $u v$ of $L$ having the following properties: (1) the set $A+a p-p$ contains $u$ and $B+b p-p$ contains $v$ of $u v$, where $a p+p b=a b$, and $A+B+a b$ contains only these points of $u v$, and (2), unless $u v$ joins $A$ and $B$, in which case the theorem is proved, there does not exist another arc in $L$, having only its end points in $A+B+a b$, which joins $A+(a u-u)$ and $B+(b v-v)$, where $a u$ is either an arc of $a b$ or $a$, and $b v$ is either an arc of $a b$ or $b$; furthermore if $a u \neq a$ there does not exist an $\operatorname{arc}$ of $L$,

* Concerning the subsets of a plane continuous curve, Annals of Mathematics, vol. 27 (1925), pp. 29-46, Theorem V.

$\dagger$ This lemma is true if "bounded" is omitted. The sufficiency is true in $E_{n}$ but the necessity is not. See $\S \mathrm{V}$.

$\ddagger$ As shown in $\S \mathrm{V}$, "bounded" may be omitted. 
having at most one end point in $a b$, joining $u v-u$ and $A+a u-u$ nor if $b v \neq b$ does there exist such an arc joining $u v-v$ and $B+b v-v$. Let $w$ be the arc of $a b$ joining $a u$ and $b v$. Let (uv) be the set of $\operatorname{arcs}$ of $L$ such as $u v$ and (w) the corresponding arcs of $a b$. Every point of $a b-a-b$ is an interior point of one and at most of two of the arcs of $(w)$. And $a+b$ contains the limit points of the end point of the arcs of $(w)$. Since there exist but a countable number of $\operatorname{arcs}$ in $(w)$, it is readily shown that $(u v)+(w)$ contains two arcs joining $A$ and $B$, which are distinct except for possibly their end points. Hence the truth of the theorem is seen.

\section{ConNected subsets OF PERFECT CONTINUOUS CURVES}

It is known that a connected subset $N$ of a perfect continuous curve $M$ is not necessarily arc-wise connected. But for any two points $a$ and $b$ of $N$ the set $N^{\prime}$ contains an arc $a b$. Here the nature of the point set composed of the points of $N$, which are also on $\operatorname{arcs} a b$ of $N^{\prime}$, is considered. It is shown that this set must be connected. Some preliminary lemmas will be proved.

THEOREM 4. In order that a bounded continuous curve $M$ in $E_{2}{ }^{*}$ be perfect it is necessary and sufficient, if $p, a$, and $b$ be any three points of any subcontinuum $N$ of $M$ such that there does not exist a point distinct from $p$ which is contained in every arc of $N$ joining $p$ and $a+b$, that there exist an arc apb of $N$.

By means of Lemma 5, using a device similar to that used in the proof of Theorem 2, it is seen that the condition is sufficient. It is also necessary. For by Theorem 3 there exist two arcs of $N$ joining $p$ and $a+b$ which are either distinct, except for $p$, or form a simple closed curve, $J$ say, containing $p$ and a point of $a+b$. If they are distinct, they form an $\operatorname{arc} a p b$ of $N$; and if they are not distinct, since $N$ contains an arc $t$ joining $p$ and $a+b-J \times(a+b)$ such that $t \times(a+b) \neq a+b$, it is seen that $J+t$ contains an arc $a p b$ of $N$.

Lемма 7. If $p, a$, and $b$ are any three distinct points of a bounded, $\dagger$ perfect, continuous curve $M$ in $E_{2}$, and if $(p)$ is the set of points, each of which is on every arc of $M$ joining $p$ and $a+b$, then there exists an arc of $M$ which joins $a$ and $b$ and contains a certain point $p_{1}$ of $(p)$ and every arc of $M$ joining $a$ and $b$ contains at most $p_{1}$ of $(p)$.

As $(p)$ is closed it is seen that there exists a first point of $(p)$ on an arc of $M$, and so on every arc of $M$, joining $p$ and $a+b$. And this point, $p_{1}$ say, has the property that there exists no other point common to every arc of $M$ for $E_{n}$

* In $\S \mathrm{V}$ it will be shown that "bounded" may be omitted here and that the necessity is true

$\dagger$ As the proof depends entirely upon Theorem 4, "bounded" can be omitted and the theorem stated for $E_{n}$. 
joining it and $a+b$. Hence by Theorem 4 there exists in $M$ an $\operatorname{arc} a p_{1} b$. And it is seen that every arc of $M$ joining $a$ and $b$ contains at most this point of $(p)$.

LEMma 8. In order that a bounded* continuous curve $M$ in $E_{2}$ be perfect it is necessary and sufficient that, if $N$ is any connected subset of $M, a$ and $b$ are any two points of $N, L$ is a subset of $N^{\prime}$ consisting of all points contained in arcs $a b$ of $N^{\prime}, Q=L \times N, p$ is a point of $N-Q$, and $(p)$ is the set of all points common to every arc of $N^{\prime}$ joining $p$ and $a+b$, then there exists a point $p_{1}$ of $(p) \times Q$ and $a$ subset $W$ of $N^{\prime}-p_{1}$, which contains $p$ and is such that $W \times(a+b)=0$, $N^{\prime}-p_{1}=W+\left(N^{\prime}-p_{1}-W\right)$ separate, and every point of $W$ can be joined to $p_{1}$ by an arc of $W+p_{1}$.

In showing that the condition is necessary it is seen at once, by means of Lemma 7 , that the point $p_{1}$ and an arc $a p_{1} b$ exist. Also there exists a set $W$ composed of the points of $N^{\prime}$ which can be joined to $p_{1}$ by an arc but cannot be joined to $a+b$ by an arc of $N^{\prime}$ which does not contain $p_{1}$. As $N^{\prime}-p_{1}$ is an open subset of $N^{\prime}$, since $N^{\prime}-p_{1}$ does not contain an arc joining $p$ and $a+b$, it does not contain a connected subset which joins $p$ and $a+b$. $\dagger$ Hence $p$ and $a+b$ are separated in the weak sense and so in the strong sensef in $N^{\prime}$. Thus the condition is necessary. That it is sufficient follows from Lemma 5.

THEOREM 5. In order that a bounded continuous curve $M$ in $E_{2} \S$ be perfect it is necessary and sufficient that, if $N$ is any connected subset of $M$ of which a and $b$ are any two points, $L$ is the point set consisting of the arcs ab of $N^{\prime}$, and $Q=L \times N$, then $Q$ is a non-vacuous connected point set.

To show that the condition is necessary it is seen that, since $N^{\prime}$ is a continuous curve, $L$ and so $Q$ is a non-vacuous set. Assume that $Q=Y+Z$ separate. By Lemma 8 , for each point $p$ of $N-Q$, there exists a point $p_{1}$ and a subset $W$ of $N^{\prime}-p_{1}$ such that $N^{\prime}-p_{1}=W+\left(N^{\prime}-p_{1}-W\right)$ separate, where $W+p_{1}$ contains an arc $p p_{1}$. Thus sets $(W)$ and $\left(p_{1}\right)$ are obtained. And $(W)=(W)_{1}+(W)_{2}$, where a set $W$ of $(W)_{1}$ is such that the corresponding $p_{1}$ of $\left(p_{1}\right)$ is in $Y$ and otherwise $W$ is in $(W)_{2}$. Let $E=Y+(W)_{1}$ and $F=Z+(W)_{2}$. Then $N=Q+(W) \times N=F \times N+F \times N$. It is possible to show then that $N=E \times N+F \times N$ separate, which is a contradiction. And that the condition is sufficient is seen by assuming that it is not, and so obtaining by Lemma 5

* "Bounded" can be omitted and the necessity proved for $E_{n}$. loc. cit.

$\dagger$ R. L. Wilder, Characterizations of continuous curves that are perfectly continuous, Theorem 3,

$\ddagger$ R. L. Wilder, $A$ characterization of continuous curves by a property of their open subsets, Fundamenta Mathematicae, vol. 11, pp. 127-131, Lemma 2.

$\S$ "Bounded" may be omitted, as seen in $\S \mathrm{V}$, the necessity holds in $E_{n}$, as Lemma 8 does, and the sufficiency holds in $E_{n}$, as may be seen from the Moore-Wilder lemma. 
$N=a_{1} b_{1}+x_{1} y_{1}+x_{2} y_{2}+\cdots+b_{2}$, letting $a=a_{1}$, and $b=b_{2}$, and thus obtaining a contradiction

\section{Additional THEOREMS}

The main problem considered here is the following: if $N$ is a connected subset of a perfect continuous curve $M$, when does there exist an $\operatorname{arc}$ of $N$ joining two points of $N$ ? Also a property is obtained of a set of $\operatorname{arcs}$ of $M$ which join two distinct closed point sets.

THEOREM 6. In order that a bounded ${ }^{*}$ continuous curve $M$ in $E_{2}$ be perfect it is necessary and sufficient, if $L$ is the point set consisting of the points of any set $\dagger$ of arcs of $M$ joining any two distinct closed point sets $A$ and $B$, that every point $p$ of $L^{\prime}$ be contained in an arc of $L+p$ joining $A$ and $B$.

That the condition is necessary follows from a theorem by H. M. Gehman $\ddagger$ and that it is sufficient is seen by means of the Moore-Wilder lemma. $\S$

TheOREM 7. In order that a bounded $\|$ continuous curve $M$ in $E_{2}$ be perfect it is necessary and sufficient, if $a$ and $b$ are any two points of any connected subset $N$ of $M$ such that $N^{\prime}$ contains but a countable number of possible arcs ab, that $N$ contain an arc $a b$.

The condition is necessary. For assume that $N$ does not contain an arc $a b$. Then every arc $a b$ of $N^{\prime}$ contains a point of $N^{\prime}-N$. Since these arcs are countable in number, let $(z)$ be a set of points obtained by taking a point of $N^{\prime}-N$ from each arc $a b$ of $N^{\prime}$. For each point $z$ of $(z), N^{\prime}-z$ is an open subset of $N^{\prime}$, and there exist a countable infinity of such sets. As $N$ is common to all these open sets, it is contained in the quasi-open subset, $Q$, of $N^{\prime}$ determined by this countable infinity of open subsets of $N^{\prime}$. But as $Q$ is arc-wise

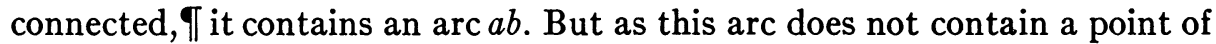
(z) a contradiction has been obtained.

The sufficiency follows from Lemma 5 .

\section{Note}

Many of the theorems of this paper have been stated as holding for a bounded continuous curve. This is due to the fact that a theorem** by H. M.

* As shown in $\S \mathrm{V}$ "bounded" may be omitted here; the sufficiency holds in $E_{n}$ but the necessity does not.

$\dagger$ It is to be noted that this set is not necessarily the set of all $\operatorname{arcs}$ of $M$ joining $A$ and $B$.

$\ddagger$ Loc. cit., Theorem 5 .

$\S$ R. L. Wilder, Concerning continuous curves, Fundamenta Mathematicae, vol. 7 (1927), p. 371, Lemma 1.

|| Bounded may be omitted here. The necessity holds for any locally compact metric space.

T R. L. Wilder, Characterizations of continuous curves that are perfectly continuous, loc. cit., Theorem 3.

** Loc. cit., Theorem 5 . 
Gehman is used in the proofs, which is known to be untrue for unbounded continuous curves. However the following lemma could have been used everywhere instead, and so the theorems of this paper hold whether the continuous curve is bounded or not.

LEMma. In order that a continuous curve $M$ in $E_{2}$ be perfect it is necessary and sufficient that $M$ does not contain an infinite sequence of distinct subcontinua with two sequential limit points.*

The sufficiency is evident from the Moore-Wilder lemma which is known to hold for the unbounded case in $E_{n}$. And the necessity is proved as follows: let $a$ and $b$ be the sequential limit points referred to in the theorem. By a well known theorem $\dagger$ there exists in $M$ a bounded continuous curve $K$ containing $a$ and all points of $M$ in a certain neighborhood of $a$, but not containing $b$. It is clear that there will exist then points of infinitely many of the given subcontinua that are not in $K$. Consequently, since $K$ is perfect,the theorem of Gehman is violated by portions of the given continua that lie in $K$.

It is known from an example by G. T. Whyburn $\ddagger$ that neither the theorem by $H$. M. Gehman nor the above lemma is true for $E_{3}$. This example further shows that neither the necessity in Theorem 6 nor Lemma 6 is true for $E_{3}$.

The necessity of the following theorem is seen by means of a theorem by W. L. Ayres $\S$ and the sufficiency is true because of the proof of Theorem 2 . The necessity holds for $E_{n}$ and the sufficiency for $q>2$.

TheOREM 3'. Let $q=2$. Then in order that a continuous curve $M$ in $E_{2}$ be perfect it is necessary and sufficient, if $L$ is the point set consisting of the points of any set of arcs of $M$ joining any two distinct closed point sets $A$ and $B$, that either there exist a set $N$ of $q-1$ points of $L^{\prime}-A-B$ such that every arc of $L^{\prime}$ joining $A$ and $B$ contains at least one point of $N$ or there exist at least $q$ arcs of $L^{\prime}$, distinct except for possibly their end points, joining $A$ and $B$.

It is seen that the proof of Theorem 4 will go through for $E_{n}$, if the necessity of the above theorem, which is true for $E_{n}$, is used in place of Theorem 3 there.

${ }^{*}$ A point $q$ is said to be a sequential limit point of a set of continua $C_{i}(i=1,2, \cdots)$ if every region containing $q$ contains points of all except a finite number of the sets $C_{i}$.

$\dagger \mathrm{H}$. Hahn, Mengentheoretische Characterisierung der stetigen Kurve, Wiener Sitzungsberichte, vol. 123 (Part IIa), pp. 2433-2489; see Theorem XXI, p. 2475.

$\ddagger$ Bulletin of the American Mathematical Society, vol. 34 (1928), p. 551. This paper is to appear in Mathematische Annalen. The example has been given in a paper by R. L. Wilder, Proceedings of the National Academy of Sciences, vol. 16 (1930), p. 234. Using the notation of Professor Wilder to show that Theorem 6 does not hold in $E_{3}$, let $L=N, a=A, b=B$, and $p=\left(0,0,2^{1 / 2} / 2\right)$. Then there does not exist an arc $a p b$ of $L+p$. Similarly Lemma 6 is shown to be untrue in $E_{3}$.

$\S$ Loc. cit., Theorem 6.

UNIVERSity OF Michigan, Ann Arbor, Mich. 